\title{
Study on propagation of pulse current discharges in plant tissue
}

\author{
Nusratillo Tashpulatov ${ }^{1 *}$, Odina Nazarova $^{2}$, Dilafruz Yuldosheva $^{2}$, Azamat Tabaev $^{1}$, and Roza Amanboeva ${ }^{3}$ \\ ${ }^{1}$ Tashkent Institute of Irrigation and Agricultural Mechanization Engineers, 100000 Tashkent, Uzbekistan \\ ${ }^{2}$ Research Institute of Forestry, 111104 Tashkent, Uzbekistan \\ ${ }^{3}$ Gulistan State University, 120100 Gulistan, Uzbekistan
}

\begin{abstract}
This article provides a theoretical description of the propagation of current pulses in plant tissue and the processes of destruction of cellular structures of harmful pathogens in the plant organism. Plants, from the point of view of electricity, are regarded as a well-conductive cable. The peel of the plant is the sheath of the cable, the fire (core) is the electrically conductive conductor. When infected with diseases, the growth of the cellular structure occurs and, as a result, the resistance of the plant tissue decreases. When current is applied, it passes through the circuit with the least resistance and thus provides the lethal effect of harmful microorganisms.
\end{abstract}

\section{Introduction}

Electrical effects on plants can be used to encourage their growth, development, and productivity, as well as to cause deadly harm for ripening, drying, increasing juice output, eliminating weeds, and so forth [1-4]. This action can be carried out by a variety of electric and magnetic fields, different types and stages of electrical discharges, and various electric currents, such as DC pulsed, alternating sinusoidal, non-sinusoidal AC with and without an electric spark discharge, and so on $[5,6]$. It is crucial to determine the most valuable of these currents from an energy and technology standpoint.

Depending on the type and method of growing and fruiting, cultivated plants are very diverse in structure. Therefore, the impact on plant organisms requires a thorough study of their cellular structures [7-9]. As a rule, perennial plants and trees have a tighter and stronger cellular structure [10]. This feature allows them to resist certain viruses and diseases. Plants of vegetable and melon crops, in comparison with them, have, on the contrary, a less dense cellular structure [1113]. Their peel and shives consist mainly of moisture, through which nutrients are transferred to all parts of the plant organism. When evaluated in terms of electricity, these plants can be regarded as a well-electrically conductive cable. The peel is the sheath of the cable, the shives (core) is the electrically conductive conductor. If the plant organism is infected with the larvae of worms of pathogenic nematodes, then, according to the known features of the nematodes, liquid-filled swellings are formed in the infected areas of plant material [14]. In these areas of a diseased plant, as is known, the cell membranes are upset [2]. For this reason, for theoretical study and creation of diseased and healthy plants as a material for processing, they can be considered as a vessel filled with a variety of liquid. Our goal was to theoretically study the propagation of high-voltage pulsed current over the infected plant areas and the mechanism of propagation of high-voltage pulses.

\section{Theory and Methods}

As a rule, instantaneous forces are called forces acting during a negligible time interval " $\tau$ ", to which high-voltage pulses of voltage and current can be attributed, which have a finite value and which can be described by the following general formula [4]:

$$
S=\lim _{\tau \rightarrow 0}(P \bullet \tau)
$$

Usually, such pulses arise, for example, when molecules collide in a liquid medium.

To study the vibrations caused by these pulses, we use the general solution of the differential equation of forced vibrations under the initial conditions by the following formula [3]:

$$
\frac{d \Pi \Pi}{\partial q_{j}}=\mathbf{O} \quad(j=1,2, \ldots \ldots ., S)
$$

${ }^{*}$ Corresponding author: nusratillo@mail.ru 
where: $\Pi$ - energy pulse

$S$ - pulse number

$$
q=\frac{1}{a k^{*}} \int_{0}^{t} Q_{F}\left(t_{1}\right) e^{-n\left(t-t_{1}\right)} \sin k^{*}\left(t-t_{1}\right) d t_{1}
$$

where: $k^{*}$ - damped-vibration frequency.

Let us first of all find the equation of vibrations caused by a constant generalized force $Q_{F}$, suddenly applied to the system at a time $t_{1}=0$ and valid for a certain period of time $\tau_{t}$. By equating formula (2), we get:

$$
\begin{aligned}
& q=\frac{Q_{F}}{a k^{*}} \int_{0}^{t} e^{-n\left(t-t_{1}\right)} \sin k^{*}\left(t-t_{1}\right) d t_{1}= \\
& =\frac{Q_{F}}{a k^{*}} \frac{k^{*}}{n^{2}+\left(k^{*}\right)^{2}}\left\{1-e^{-n t}\left(\frac{n}{k^{*}} \sin k^{*} t+\cos k^{*} t\right)\right\}
\end{aligned}
$$

or

$$
q=\frac{Q_{F}}{c}\left\{1-e^{-n t}\left(\frac{n}{k^{*}} \sin k^{*} t+\cos k^{*} t\right)\right\}_{t \leq \tau}
$$

where, $Q_{F}$ - forces acting by voltage pulses.

Vibrations determined by equation (4) exist as long as the force acts $Q_{F}$ i.e. if $t \leq \tau$, i.e. current pulse occurs.

Find the maximum value of the coordinate $q=q(t)$. From equation (4) we have:

$$
q=\frac{Q_{F}}{c}\left\{-e^{-n t}\left(n \cos k^{\bullet} t-k^{\bullet} \sin k^{\bullet} t\right)+n e^{-n t}\left(\frac{n}{k^{*}} \sin k^{\bullet} t+\cos k^{*} t\right)\right\}
$$

By equating the generalized speed of the pulse current to zero, we get

$$
\mathrm{e}^{-\mathrm{nt}} \sin \mathrm{k}^{*} \mathrm{t}=0 \text {, }
$$

which for a finite value of $t$ leads to the condition

$$
\sin \mathrm{k}^{*} \mathrm{t}=0 ;, \mathrm{t}=\pi / \mathrm{K}^{\bullet}
$$

since at $t=0, q_{o}=0$ consequently, the maximum value of $\boldsymbol{q}$ reaches at

$$
t=\frac{T^{*}}{2}
$$

i.e. in that case, when time interval $t$ is equal to half the period of damped-vibrations. $\left(T^{*}\right)$ Maximum value of " $q$ " equals:

$$
q_{\max }=\frac{Q_{F}}{c}\left(1+e^{-\frac{n T^{*}}{2}}\right)
$$

Since under the force static action $\mathrm{Q}_{\mathrm{F}}$

$$
q_{c}=\frac{Q_{F}}{c}
$$

then the dynamic factor for the considered case is $\left(\tau \geq \frac{T^{*}}{2}\right)$

This factor is always greater than one.

$$
\lambda=1+e^{-\frac{n T^{*}}{2}}
$$

For the case $\mathrm{n}=0$ (resistance not considered) equation (4) takes the following form:

$$
q_{c}=\frac{Q_{F}}{c}(1-\cos k t)_{t \leq \tau}
$$

The dynamic effect of a suddenly applied constant force (shock wave of a pulsed current) in this case, according to formula (6), is twice the static $\lambda=2$.

The equation of vibrations of the system after the termination of the action of the force (the passage of the impulse current is stopped) $(t \geq \tau)$ can be obtained as follows: 
generalized force equal to $-\mathrm{Q}_{\mathrm{F}}$, applied to the system at a point in time $t=\tau$, causes vibrations determined by an equation similar to equation (4), namely:

$$
q=-\frac{Q_{F}}{c}\left\{1-e^{-n(t-\tau)}\left[\frac{n}{k^{*}} \sin k^{*}(t-\tau)+\cos k^{*}(t-\tau)\right]\right\}
$$

By summing up these vibrations with the vibrations caused by the force + QF applied to the system at the moment of time $t=0$, we get the following vibration equation for the case $t \geq \tau$, where $\tau$ - force action duration $+Q_{F}$ :

$$
\begin{aligned}
& q=-\frac{Q_{F}}{c}\left\{e^{-n(t-\tau)}\left[\frac{n}{k^{*}} \sin k^{*}(t-\tau)+\cos k^{*}(t-\tau)\right]-\right. \\
& \left.-e^{-n t}\left(\frac{n}{k^{*}} \sin k^{*} t+\cos k^{*} t\right)\right\}
\end{aligned}
$$

If,

at $\mathrm{t} \geq \tau$

$$
\tau<\frac{T^{*}}{2}
$$

then the generalized coordinate $\mathrm{q}$, determined by equation (7), reaches its maximum value after the cessation of the action of the force, i.e. at some point in time $t_{m}>\tau$.

This moment in time can be determined from the equation,

$$
\begin{gathered}
\dot{q}=0 \\
e^{-n t} m\left\{\sin k^{*} t_{m}-e^{n \tau} \sin k^{*}\left(t_{m}-\tau\right)\right\}=0 \\
\operatorname{tg} k^{*} t_{m}=\frac{\sin k^{*} \tau}{\cos k^{*} \tau-e^{-n \tau}}
\end{gathered}
$$

Relation (8) allows obtaining the following maximum value $q$ :

$$
q_{\max }=\frac{Q_{F} e^{-n t_{m}}}{c}\left\{e^{n \tau} \cos k^{*}\left(t_{m}-\tau\right)-\cos k^{*} t_{m}\right\}
$$

In this case, the dynamic factor is:

$$
\lambda=e^{-n t} m\left\{e^{n \tau} \cos k^{*}\left(t_{m}-\tau\right)-\cos k^{*} t_{m}\right\}
$$

If value $« n »$ much less than « $k »$, then the resistance can be neglected. All formulas in that case $(n=0)$ simplify and take this form:

$$
\begin{aligned}
q=\frac{Q_{F}}{c}\{\cos k(t-\tau) & -\cos k t\}=\frac{2 Q_{F}}{c} \sin \frac{k \tau}{2} \sin k\left(t-\frac{\tau}{2}\right) \\
t_{m} & =\frac{\pi}{2 k}+\frac{\tau}{2} ; \\
q_{\max } & =\frac{2 Q_{F}}{c} \sin \frac{k \tau}{2} ; \\
\lambda & =2 \sin \frac{k \tau}{2} .
\end{aligned}
$$

\section{Results}

Depending on the duration of the action of a constant force suddenly applied to the system (the strength of the shock of the impulse current) $Q_{F}$, factor $\lambda$, defined by formulas (9) and (10), can be more and less than unity [1].

To study vibrations caused by a current pulse of instantaneous strength 


$$
S=\lim \left(Q_{F} \cdot l\right)_{\tau \rightarrow 0}
$$

we represent the previous equation in the following form:

$$
q=\frac{Q_{F} * \tau}{c} \times \frac{e^{-n(t-\tau)}\left\{\frac{n}{k^{*}} \sin k^{*}(t-\tau)+\cos k^{*}(t-\tau)\right\}-e^{-n t}\left(\frac{n}{k^{*}} \sin k^{*} t+\cos k^{*} t\right)}{\tau}
$$

The right side of this expression for $\tau \rightarrow 0$ turns into an uncertainty of the form $\frac{0}{0}$. Revealing this uncertainty, we get:

$$
\begin{array}{r}
q=\frac{S^{*} e^{-n t}}{c} \frac{k^{2}}{k^{*}} \sin k^{*} t \\
q=\frac{S}{a k^{*}} e^{-n t} \sin k^{*} t
\end{array}
$$

Maximum value of $\boldsymbol{q}$ at the same time equal:

$$
q_{\max }=\frac{S}{a k^{*}} e^{-n t_{m}} \sin k^{*} t_{m}
$$

where:

$$
t_{m}=\frac{1}{k^{*}} \operatorname{arctg} \frac{k^{*}}{n}
$$

For low damped factor «n» at $k^{*} \approx k$

$$
\begin{aligned}
& t_{m} \approx \frac{T}{4}=\frac{\pi}{2 k} \\
& q_{\max } \approx \frac{S}{a k} e^{-\frac{n T}{4}}=\frac{S}{a k} e^{-\frac{\pi n}{2 k}}
\end{aligned}
$$

If $n=0$, then

$$
\begin{gathered}
q=\frac{S}{a k} \sin k t=\frac{S k}{c} \sin k t \\
q_{\max }=\frac{S k}{c}
\end{gathered}
$$

Value $S k$ can be viewed as an equivalent generalized force $Q_{\text {eqv }}$, the static action of which determines the maximum deviation of the system from its equilibrium position, caused by $S$.

Consider vibrations caused by instantaneous current impulses. If impulses arise after a period of time then:

$$
t_{j}(j=1,2, \ldots \ldots, s)\left(t_{1}=0\right)
$$

where, $S$ - pulse number, then the equation of forced vibrations at $t>t_{l}$ has a form as follows:

$$
q=\frac{1}{a k^{*}} \sum_{j=1}^{S} S_{j} e^{-n\left(t-t_{j}\right)} \sin k^{*}\left(t-t_{j}\right)
$$

For equal impulses $\left(S_{j}=S\right)$ and equal time $\operatorname{span}\left(t_{j}=j_{\tau}\right)$

If the time interval between the arising impulses

$$
q=\frac{S}{a k^{*}} \sum_{j=0}^{S-1} e^{-n(t-j \tau)} \sin k^{*}(t-j \tau) \text { at } \mathrm{t}>\mathrm{j} \tau
$$

$$
\tau=\frac{2 \pi}{k^{*}}=T^{*}
$$


then the so-called shock resonance of the current occurs.

In that case:

$$
\left.q=\frac{S}{a k^{*}} \sum_{j=0}^{S=1} e^{-n\left(t-j T^{*}\right)} \sin k^{*}\left(t-j T^{*}\right) \text { at } t\right\rangle(s-l) T^{*}
$$

Taking as a start the countdown moment

$$
t=(s-l) T^{*}
$$

we get

$$
q=\frac{S}{a k^{*}} e^{-n t} \sin k^{*} t\left\{e^{-(s-1) n T^{*}}+e^{-(s-2) n T^{*}}+\ldots+e^{-n t^{*}}+1\right\}
$$

or

$$
q=\frac{S\left(1-e^{-s n T^{*}}\right)}{a k^{*}\left(1-e^{-n T^{*}}\right)} e^{-n t} \sin k^{*} t
$$

If $\mathrm{n}=0$ current pulse number.

For an unlimited number of periodically arising impulses of instantaneous forces $(s \rightarrow \infty)$ equation (24) takes the following form:

$$
\begin{gathered}
q=\frac{S * s}{a k} \sin k t \\
q=\frac{S}{a k^{*}\left(1-e^{-n T^{*}}\right)} e^{-n t} \sin k^{*} t
\end{gathered}
$$

This equation is applicable for the time interval:

$$
\mathrm{O} \leq t \leq T
$$

For the next intervals, the cycle is repeated, i.e. the motion of the system is determined by the same equation. The vibration graph is shown in Fig. 1.

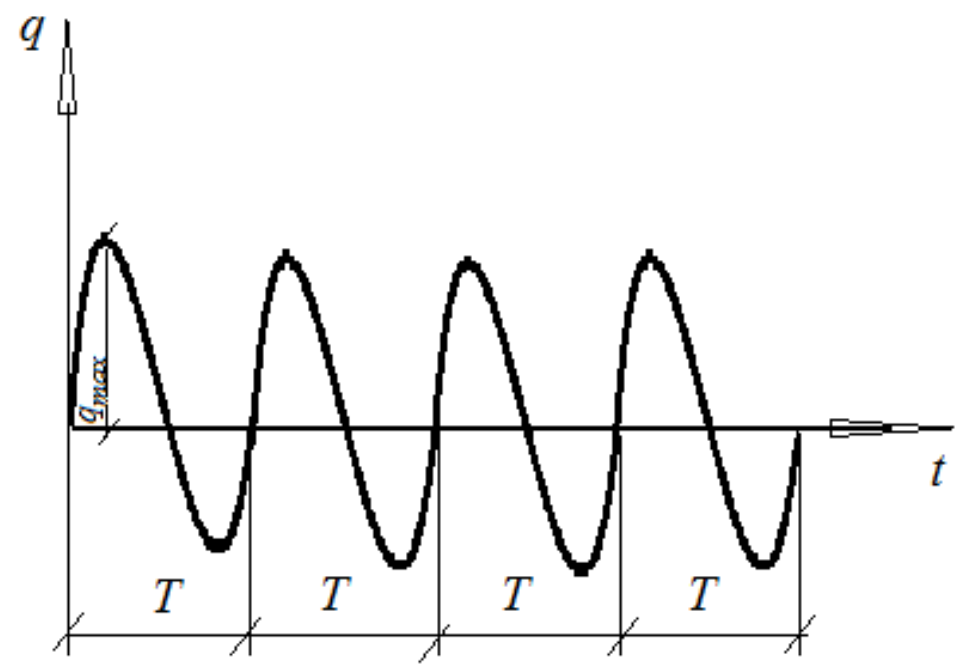

Fig.1. Pulse vibration graph under the influence of periodically disturbing forces

With no resistance $(n=0)$ amplitude of vibrations $q_{\max } \rightarrow \infty$, i.e. with an increase in the number of pulses according to formula (25), the amplitude increases indefinitely. 
The graph of the growth of these fluctuations is shown in Fig. 2.

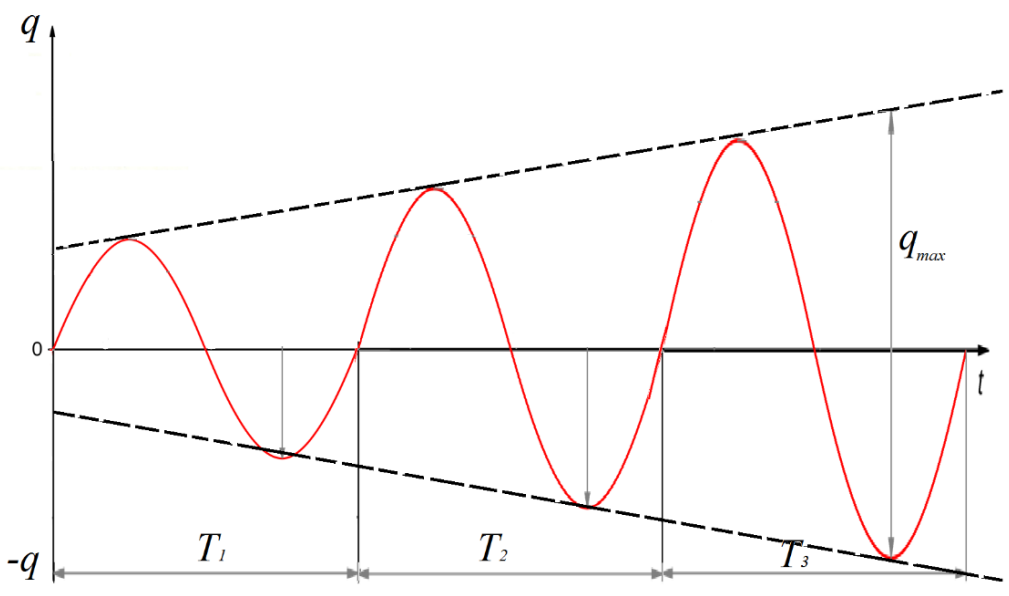

Fig.2. The graph of vibrations arising from alternating current forces

Thus, in the presence of shock resonance, as well as in the case of resonance resulting from the action of periodic disturbing forces due to the impact of a pulsed current, it is necessary to take into account even insignificant resistance forces characterized by a small damping factor. Vibrations caused by alternating pulses arising at intervals of time $\tau=\frac{T^{*}}{2}$, can be investigated in a similar way.

The motion of the system in this case is determined by the equation

$$
\left.q=\frac{S}{a k^{*}} \sum_{j=0}^{s-1}(-1)^{j} e^{-n\left(t-j \frac{T^{*}}{2}\right)} \sin k^{*}\left(t-j \frac{T^{*}}{2}\right) \text { at } t\right\rangle(s-l) \frac{T^{*}}{2}
$$

For a finite number of pulses from the time $t=(s-l) \frac{T^{*}}{2}$ this equation can be represented in the following form:

$$
q=\frac{S\left(1-e^{-0,5 s n T^{*}}\right)}{a k^{*}\left(1-e^{-0,5 n T^{*}}\right)} e^{-n t} \sin k^{*} t
$$

If the number of pulses $s \rightarrow \infty$, the equation (28) takes the following form:

$$
q=\frac{S e^{-n t}}{a k^{*}\left(1-e^{-0,5 n T^{*}}\right)} \sin k^{*} t \quad \text { at } \quad 0 \leq t \leq T .
$$

For a finite number of pulses and a damping factor $n=0$ by the equation we get the uncertainty of the form $\frac{0}{0}$. By expanding this uncertainty according to the general rule, we get

$$
q=\frac{S * s}{a k} \sin k t
$$

Fig. 3 shows a graph of the growth of these vibrations with an increase in the number of shock current pulses, «s». This is due to the fact that with an increase in the number of pulses in liquid-filled vessels (such as the infected areas of plants), a resonant increase in the amplitude of subsequent pulses can occur. Through a measured increase in the amplitude of the impulses, they can have insignificant effectiveness in the destruction of microscopic membranes, such as the egg cell structure of nematode helminth

Therefore, we believe that the best efficiency of the understanding of cells can be achieved with the parameters of pulsed discharges having, according to the formula 25 and the harmonic amplitude shown in Fig. 2. 


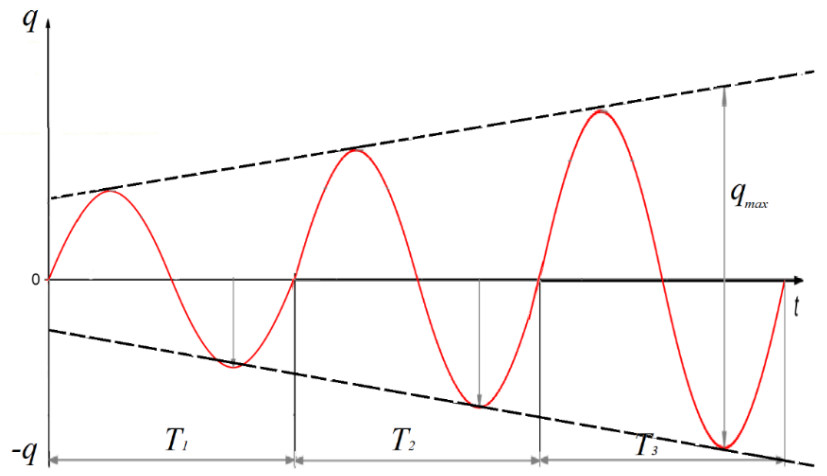

Fig.3. Graph of the increase in vibrations due to the resonance of the shock current pulses

\section{Conclusions}

1. The results of theoretical studies have proven that it is possible to achieve positive results using a high-voltage pulsed current when killing nematode larvae and worms.

2. The main active factor in the defeat of cysts, larvae and nematode worms found in plant tissues is the electrohydraulic impact force of high voltage pulses.

3. The use of such technology in agriculture can eliminate pollution of the environment, soil, food products of agriculture.

4. This technique allows you to save resources, extraordinary treatments, labor time and strength, technology, as well as various chemicals with all the ensuing negative consequences.

\section{References}

1. N.T. Tashpulatov, Method of harvesting plants. A.S. No. 3456 certifying 504 registered in the state. register of inventions, industrial designs and utility models of the Republic of Uzbekistan, Tashkent (1996)

2. T. Toshpulatov, O. Tursunov, D. Kodirov, G. Kholmuratova, Environmentally friendly technology for the destruction of tobacco mosaic viruses (TMV) from selected species of plants, IOP Conf. Ser.: Earth Environ. Sci. 614, 012133 (2020)

3. M.I. Ismailov, N.T. Tashpulatov, E.O. Bozorov, Zh.N. Tashpulatov, Features of a pulse discharge during electrical pulse processing of plant materials and problems associated with wear of processing electrodes, The Republican Scientific and Practical Conference on the Problems of Efficient Use of Energy in Agro-industrial Complexes, Tashkent (2001)

4. S.P. Strelkov, Introduction to the theory of vibrations, Science, Moscow (1989)

5. N. Toshpulatov, O. Tursunov, D. Kodirov, A. Maksumkhanova, Z. Yusupov, Study on issues of uninterrupted power supply, energy-saving and improving the quality of electrical energy of water facilities, IOP Conf. Ser.: Earth Environ. Sci. 614, 012025 (2020)

6. A. Anarbaev, A. Muxammadiev, S. Umarov, O. Tursunov, D. Kodirov, S. Khushiev, F. Muhtarov, S. Muzafarov, J. Izzatillaev, Mobile installations for electro treatment of soils and plants with the use of photovoltaic systems as power supply, IOP Conf. Ser.: Earth Environ. Sci. 614, 012046 (2020)

7. J.W. Dobrowolski, O. Tursunov, O. Pirimov, O.J. Nazarova, Laser Biotechnology for Nutritional Health, Sustainable Environment and Development, IOP Conf. Ser.: Earth Environ. Sci. 614, 012108 (2020)

8. J.W. Dobrowolski, D. Bedla, T. Czech, F. Gambus, K. Gorecka, W. Kiszcak, T. Kuzniar, R. Mazur, A. Nowak, M. Sliwka, O. Tursunov, A. Wagner, J. Wieczorek, M. Swiatek, Integrated Innovative Biotechnology for Optimization of Environmental Bioprocesses and a Green Economy, Optimization and Applicability of Bioprocesses, eds H. Purohit, V. Kalia, A. Vaidya, A. Khardenavis, Springer, Singapore, chapter 3, pp. 27-71 (2017)

9. J.W. Dobrowolski, J. Kobylarczyk, O. Tursunov, S.Q. Toh, Integration of Local Eco-Innovation with Global Problems of Protection of the Natural Environment and Bio-Based Green Economy, In Proceedings : AASRI International Conference on Circuits and Systems (CAS), Atlantis Press 9, 25-28 (2015)

10. P. E. Lauri, J. J. Kelner, C. Trottier, E. Costes, Insights into secondary growth in perennial plants: its unequal spatial and temporal dynamics in the apple (Malus domestica) is driven by architectural position and fruit load, Annals of Botany 105(4), 607-616, (2010)

11. A. K. Yadav, S. V. Singh, Osmotic dehydration of fruits and vegetables: a review, Journal of Food Science and Technology 51(9), 1654-1673 (2014) 
12. M. Asaduzzaman, M.R. Talukder, H. Tanaka, M. Ueno, M. Kawaguchi, S. Yano, T. Ban, T. Asao, Production of Low-Potassium Content Melon Through Hydroponic Nutrient Management Using Perlite Substrate, Front. Plant Sci. 9, $1382(2018)$

13. R.M.A. Machado, R.P. Serralheiro, Soil Salinity: Effect on Vegetable Crop Growth. Management Practices to Prevent and Mitigate Soil Salinization, Horticulturae 3, 30 (2017)

14. J.T. Jones, A. Haegeman, E.G.J. Danchin, H.S. Gaur, J. Helder, M.G. K. Jones, T. Kikuchi, R. ManzanillaLópez, J.E. Palomares-Rius, W.M.L. Wesemael, R.N. Perry, Top 10 plant-parasitic nematodes in molecular plant pathology, Molecular Plant Pathology 14, 946-961 (2013) 